\title{
Finite element analysis of FRP pipelines' dynamic stability
}

\author{
D. G. Pavlou \& A. I. Nergaard \\ Department of Mechanical and Structural Engineering \\ and Materials Science, University of Stavanger, Norway
}

\begin{abstract}
A finite element model is derived for flow-induced instability analysis of multilayered, filament wound FRP pipelines resting on equispaced elastic supports. The critical flow velocity causing instability is obtained by taking into account the anisotropic properties of pipes material, the elastic properties of pipeline supports and flow parameters. A representative example is solved and discussed.
\end{abstract}

Keywords: FRP pipeline, flow induced instability, critical flow velocity, FEM.

\section{Introduction}

Even though fiber reinforced polymeric (FRP) materials, apart from high strength and low density provide superior behavior than steel in corrosion, reducing thus the maintenance cost, their use for pipeline installations is not as wide as should be expected. However, in the last decade the increasing rate of adopting FRP's for pipelines means that the industry is not far from extended applicability of composite materials for pipeline projects [1]. Since steel is still the main material used for long pipeline infrastructures, the flow-induced instability has systematically been analyzed only for such isotropic material. Application of the accumulated dynamic analysis know-how developed for steel pipelines on FRP ones is not possible due to their anisotropic behavior. In the present work, finite element (FE) analysis is carried out for critical flow velocities estimation of filament wound, multi-layered FRP pipelines resting on elastic supports. 


\section{Classical lamination theory}

Based on Kirchhoff assumption, the correlation of strains $\varepsilon_{x}^{o}, \varepsilon_{y}^{o}, \gamma_{x y}^{o}$ and curvatures $k_{x}^{o}, k_{y}^{o}, k_{x y}^{o}$ of the middle plane of a laminate (Figure 1) with the corresponding force (per unit length) and moment (per unit length) resultants $N_{x}, N_{y}, N_{x y}, M_{x}, M_{y}, M_{x y}$ is given by the following equation (e.g. [2]):

$$
\left\{\begin{array}{l}
\varepsilon_{x}^{0} \\
\varepsilon_{y}^{0} \\
\gamma_{x y}^{0} \\
k_{x}^{0} \\
k_{y}^{0} \\
k_{x y}^{0}
\end{array}\right\}=\left[\begin{array}{llllll}
a_{11} & a_{12} & a_{16} & b_{11} & b_{12} & b_{16} \\
a_{12} & a_{22} & a_{26} & b_{21} & b_{22} & b_{26} \\
a_{16} & a_{26} & \alpha_{66} & b_{61} & b_{62} & b_{66} \\
b_{11} & b_{12} & b_{61} & d_{11} & d_{12} & d_{16} \\
b_{12} & b_{22} & b_{62} & d_{12} & d_{22} & d_{26} \\
b_{16} & b_{26} & b_{66} & d_{16} & d_{26} & d_{66}
\end{array}\right]\left\{\begin{array}{l}
N_{x} \\
N_{y} \\
N_{x y} \\
M_{x} \\
M_{y} \\
M_{x y}
\end{array}\right\}
$$

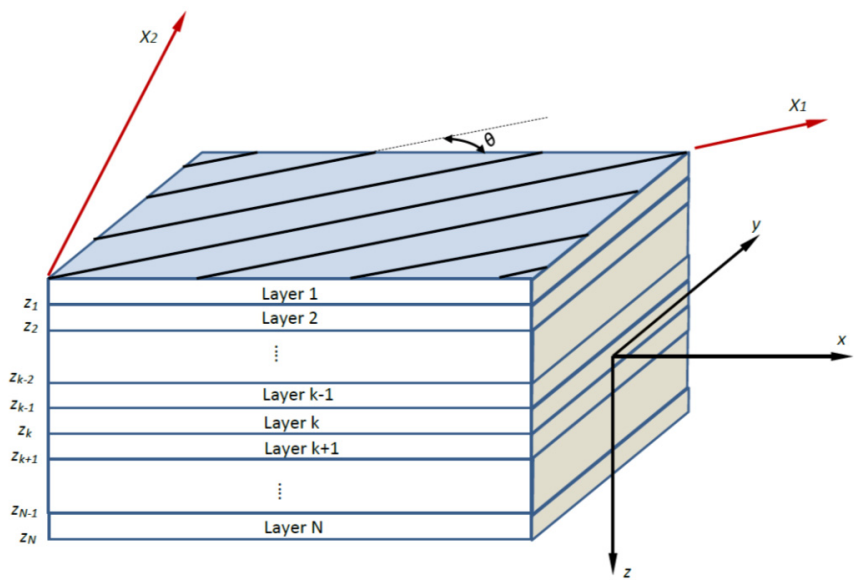

Figure 1: Layout of an FRP laminate.

The components $a_{i j}, b_{i j}, d_{i j}$ of the above matrix can be derived by the following equation:

$$
\left[\begin{array}{llllll}
a_{11} & a_{12} & a_{16} & b_{11} & b_{12} & b_{16} \\
a_{12} & a_{22} & a_{26} & b_{21} & b_{22} & b_{26} \\
a_{16} & a_{26} & \alpha_{66} & b_{61} & b_{62} & b_{66} \\
b_{11} & b_{12} & b_{61} & d_{11} & d_{12} & d_{16} \\
b_{12} & b_{22} & b_{62} & d_{12} & d_{22} & d_{26} \\
b_{16} & b_{26} & b_{66} & d_{16} & d_{26} & d_{66}
\end{array}\right]=\left[\begin{array}{llllll}
\mathrm{A}_{11} & \mathrm{~A}_{12} & \mathrm{~A}_{16} & B_{11} & B_{12} & B_{16} \\
\mathrm{~A}_{12} & \mathrm{~A}_{22} & \mathrm{~A}_{26} & B_{12} & B_{22} & B_{26} \\
\mathrm{~A}_{16} & \mathrm{~A}_{26} & \mathrm{~A}_{66} & B_{16} & B_{26} & B_{66} \\
B_{11} & B_{12} & B_{16} & D_{11} & D_{12} & D_{16} \\
B_{12} & B_{22} & B_{26} & D_{12} & D_{22} & D_{26} \\
B_{16} & B_{26} & B_{66} & D_{16} & D_{26} & D_{66}
\end{array}\right]^{-1}
$$


In equation (2) the following definitions are valid:

$$
\begin{gathered}
A_{i j}=\sum_{k=1}^{N} \bar{Q}_{i j k}\left(z_{k}-z_{k-1}\right) \\
B_{i j}=\frac{1}{2} \sum_{k=1}^{N} \bar{Q}_{i j k}\left(z_{k}^{2}-z_{k-1}^{2}\right) \\
D_{i j}=\frac{1}{3} \sum_{k=1}^{N} \bar{Q}_{i j k}\left(z_{k}^{3}-z_{k-1}^{3}\right)
\end{gathered}
$$

In the above equations, $N$ is the number of layers composing the laminate, $z_{k}$ represents the distances of any layer $k$ from the end surface of the laminate, and the parameters $\bar{Q}_{i j}$ are given for each lamina by the following equations:

$$
\begin{gathered}
\bar{Q}_{11}=Q_{11} m^{4}+2\left(Q_{12}+2 S_{66}\right) n^{2} m^{2}+Q_{22} n^{4} \\
\bar{Q}_{12}=\left(Q_{11}+Q_{22}-4 Q_{66}\right) n^{2} m^{2}+Q_{12}\left(n^{4}+m^{4}\right) \\
\bar{Q}_{16}=\left(Q_{11}-Q_{12}-2 Q_{66}\right) n m^{3}+\left(Q_{12}-Q_{22}+2 Q_{66}\right) n^{3} m \\
\bar{Q}_{22}=Q_{11} n^{4}+2\left(Q_{12}+2 Q_{66}\right) n^{2} m^{2}+Q_{22} m^{4} \\
\bar{Q}_{26}=\left(Q_{11}-Q_{12}-2 Q_{66}\right) n^{3} m+\left(Q_{12}-Q_{22}+2 Q_{66}\right) n m^{3} \\
\bar{Q}_{66}=\left(Q_{11}+Q_{22}-2 Q_{12}-2 S_{66}\right) n^{2} m^{2}+Q_{66}\left(n^{4}+m^{4}\right)
\end{gathered}
$$

The parameters $m, n$ depends on the fiber orientation angle $\vartheta$ of each lamina (Figure 1):

$$
\begin{gathered}
m=\cos \vartheta \\
n=\sin \vartheta
\end{gathered}
$$

The parameters $Q_{i j}$ are correlated to material properties in the principal coordinate system $x_{1}-x_{2}$ (Figure 1) of each individual lamina composing the laminate:

$$
Q_{11}=\frac{E_{1}}{1-v_{12} v_{21}}
$$




$$
\begin{gathered}
Q_{12}=\frac{v_{12} E_{2}}{1-v_{12} v_{21}}=\frac{v_{21} E_{1}}{1-v_{12} v_{21}} \\
Q_{22}=\frac{E_{2}}{1-v_{12} v_{21}} \\
Q_{66}=G_{12} \\
v_{21}=\frac{E_{2}}{E_{1}} v_{12}
\end{gathered}
$$

$E_{1}, E_{2}$ are the elasticity moduli, $G_{12}$ is the shear modulus and $v_{12}, v_{21}$ are the Poisson's ratios in the principal coordinate system $x_{1}-x_{2}$.

\section{Motion equation of multi-layered filament wound FRP pipes}

According to [3], the motion equation of filament wound FRP pipe is given by:

$$
S_{e q} \frac{\partial^{4} w(x, t)}{\partial x^{4}}+M U^{2} \frac{\partial^{2} w(x, t)}{\partial x^{2}}+2 M U \frac{\partial^{2} w(x, t)}{\partial x \partial t}+(M+m) \frac{\partial^{2} w(x, t)}{\partial t^{2}}=0
$$

$M, m$ is the mass per unit length for the liquid and the pipe's material respectively, $U$ is the mean velocity of the liquid, $w(x, t)$ is the elastic deflection of the pipe and $S_{e q}$ is the equivalent bending stiffness of the pipe for bending moment calculation:

$$
M(x)=S_{e q} \frac{d^{2} w}{d x^{2}}
$$

The parameter $S_{e q}$ can be obtained from [3]:

$$
S_{e q}=\frac{\pi D}{2}\left(\frac{D}{2 a_{11}}+\frac{1}{d_{11}}\right)
$$

In the above equations $M(x)$ is the bending moment, and $D$ is the diameter of the pipe.

Substituting (e.g. [4]) into equation (19) the solution (22)

$$
w(x, t)=\operatorname{Re}\left[u(x) e^{i \omega t}\right],
$$

the following equation can be obtained:

$$
S_{e q} \frac{d^{4} u(x)}{d x^{4}}+M U^{2} \frac{d^{2} u(x)}{d x^{2}}+2 M U \omega i \frac{d u(x)}{d x}-(M+m) \omega^{2} u(x)=0
$$




\subsection{Element equation of a pipe segment}

Following a standard mathematical procedure the differential equation (23) can be transformed to the following matrix differential equation of first order:

$$
\frac{d}{d x}\{Y\}=[\Omega] .\{Y\}
$$

In equation (24) the following is valid:

$$
\{Y\}=\left\{u(x) \quad u^{\prime}(x) \quad u^{\prime \prime}(x) \quad u^{\prime \prime \prime}(x)\right\}^{T}
$$

and

$$
[\Omega]=\left[\begin{array}{cccc}
0 & 1 & 0 & 0 \\
0 & 0 & 1 & 0 \\
0 & 0 & 0 & 1 \\
\frac{(M+m) \omega^{2}}{S_{e q}} & \frac{-2 M U \omega i}{S_{e q}} & \frac{-M U^{2}}{S_{e q}} & 0
\end{array}\right]
$$

Therefore, the solution of the matrix equation (24) can be written:

$$
\left\{Y_{2}\right\}=e^{[\Omega] L} \cdot\left\{Y_{1}\right\}
$$

$L$ is the length of the pipe segment $1-2$.

$$
\begin{gathered}
\left\{Y_{2}\right\}=\left\{u(L) \quad u^{\prime}(L) \quad u^{\prime \prime}(L) \quad u^{\prime \prime \prime}(L)\right\}^{T} \\
\left\{Y_{1}\right\}=\left\{u(0) \quad u^{\prime}(0) \quad u^{\prime \prime}(0) \quad u^{\prime \prime \prime}(0)\right\}^{T}
\end{gathered}
$$

Taking into account the following relations of solid mechanics:

$$
\begin{gathered}
\vartheta(x)=u^{\prime}(x) \\
\mathrm{m}(x)=S_{e q} \mathrm{u}^{\prime \prime}(x) \\
\mathrm{f}(x)=S_{e q} \mathrm{u}^{\prime \prime \prime}(x)
\end{gathered}
$$

where $\vartheta(x), m(x), f(x)$ is the slope, bending moment and shear force distribution, the matrix equation (27) can now be written in the following form: 


$$
\left\{\begin{array}{c}
u_{2} \\
\vartheta_{2} \\
m_{2} / S_{e q} \\
f_{2} / S_{e q}
\end{array}\right\}=e^{[\Omega] L}\left\{\begin{array}{c}
u_{1} \\
\vartheta_{1} \\
m_{1} / S_{e q} \\
f_{1} / S_{e q}
\end{array}\right\}
$$

Let's assume that the matrix exponential $e^{[\Omega] L}$ is expressed by the following matrix:

$$
e^{[\Omega] L}=\left[\begin{array}{llll}
c_{11} & c_{12} & c_{13} & c_{14} \\
c_{21} & c_{22} & c_{23} & c_{24} \\
c_{31} & c_{32} & c_{33} & c_{34} \\
c_{41} & c_{42} & c_{43} & c_{44}
\end{array}\right]
$$

Performing some rearrangements in eq. (33), the following expression can be obtained:

$$
[F]\left\{\begin{array}{l}
f_{1} \\
m_{1} \\
f_{2} \\
m_{2}
\end{array}\right\}=[U]\left\{\begin{array}{l}
u_{1} \\
\vartheta_{1} \\
u_{2} \\
\vartheta_{2}
\end{array}\right\}
$$

In equation (35) the following is valid:

$$
\begin{gathered}
{[F]=\left[\begin{array}{cccc}
c_{14} / S_{e q} & c_{13} / S_{e q} & 0 & 0 \\
c_{24} / S_{e q} & c_{23} / S_{e q} & 0 & 0 \\
c_{34} / S_{e q} & c_{33} / S_{e q} & 0 & -1 \\
c_{44} / S_{e q} & c_{43} / S_{e q} & -1 & 0
\end{array}\right]} \\
{[U]=\left[\begin{array}{cccc}
-c_{11} & -c_{12} & -1 & 0 \\
-c_{21} & -c_{21} & 0 & -1 \\
-S_{e q} c_{31} & -S_{e q} c_{32} & 0 & 0 \\
-S_{e q} c_{41} & -S_{e q} c_{42} & 0 & 0
\end{array}\right]}
\end{gathered}
$$

Therefore, the following equation for a segment 1-2 can be derived:

$$
\left\{\begin{array}{l}
f_{1} \\
m_{1} \\
f_{2} \\
m_{2}
\end{array}\right\}=[k]\left\{\begin{array}{l}
u_{1} \\
\vartheta_{1} \\
u_{2} \\
\vartheta_{2}
\end{array}\right\}
$$


In equation (38) we have:

$$
[k]=[F]^{-1}[U]
$$

\section{Pipeline resting on elastic supports}

Let's assume a pipeline resting on equispaced elastic supports (Figure 2). The elastic supports can be linear springs with elastic constant $k$. Since the length and the material properties of all pipe segments are equal, the equation (38) represents the element equation of each pipe element. Let's express the equation (38) for two successive nodes $i, j$ in the following form:

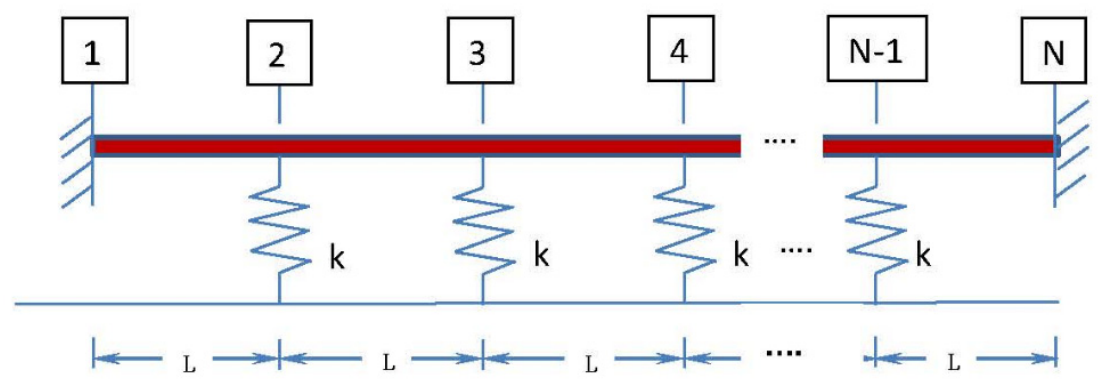

Figure 2: Nodes of a periodically multi-supported pipeline resting on elastic supports.

$$
\left\{\begin{array}{c}
f_{i} \\
m_{i} \\
f_{j} \\
m_{j}
\end{array}\right\}=\left[\begin{array}{llll}
k_{11} & k_{12} & k_{13} & k_{14} \\
k_{21} & k_{22} & k_{23} & k_{24} \\
k_{31} & k_{32} & k_{33} & k_{34} \\
k_{41} & k_{42} & k_{43} & k_{44}
\end{array}\right]\left\{\begin{array}{c}
u_{i} \\
\vartheta_{i} \\
u_{j} \\
\vartheta_{j}
\end{array}\right\}
$$

Expansion of the above equation in global coordinates for any individual element $i-j$ and superposition of the expanded element equations of all pipeline segments, yields:

$$
\left\{\begin{array}{c}
F_{1} \\
M_{1} \\
F_{2} \\
M_{2} \\
F_{3} \\
M_{3} \\
\vdots \\
F_{N-1} \\
M_{N-1} \\
F_{N} \\
M_{N}
\end{array}\right\}=\left[\begin{array}{ccccccccccc}
k_{11} & k_{12} & k_{13} & k_{14} & 0 & 0 & \ldots & 0 & 0 & 0 & 0 \\
k_{21} & k_{22} & k_{23} & k_{24} & 0 & 0 & \ldots & 0 & 0 & 0 & 0 \\
k_{31} & k_{32} & k_{33}+k_{11} & k_{34}+k_{12} & k_{13} & k_{14} & \ldots & 0 & 0 & 0 & 0 \\
k_{41} & k_{42} & k_{43}+k_{21} & k_{44}+k_{22} & k_{23} & k_{24} & \ldots & 0 & 0 & 0 & 0 \\
0 & 0 & k_{31} & k_{32} & k_{33}+k_{11} & k_{34}+k_{12} & k_{13} & k_{14} & 0 & 0 & 0 \\
0 & 0 & k_{41} & k_{42} & k_{43}+k_{21} & k_{44}+k_{22} & k_{23} & k_{24} & 0 & 0 & 0 \\
\vdots & \vdots & \vdots & \vdots & \vdots & \vdots & \vdots & \vdots & \vdots & \vdots & \vdots \\
0 & 0 & 0 & 0 & 0 & k_{31} & k_{32} & k_{33}+k_{11} & k_{34}+k_{12} & k_{13} & k_{14} \\
0 & 0 & 0 & 0 & 0 & k_{41} & k_{42} & k_{43}+k_{21} & k_{44}+k_{22} & k_{23} & k_{24} \\
0 & 0 & 0 & 0 & 0 & 0 & \ldots & k_{31} & k_{32} & k_{33} & k_{34} \\
0 & 0 & 0 & 0 & 0 & 0 & \ldots & k_{41} & k_{42} & k_{43} & k_{44}
\end{array}\right]\left\{\begin{array}{c}
u_{1} \\
\vartheta_{1} \\
u_{2} \\
\vartheta_{2} \\
u_{3} \\
\vartheta_{3} \\
\vdots \\
u_{N-1} \\
\vartheta_{N-1} \\
u_{N} \\
\vartheta_{N}
\end{array}\right\}
$$


or in an abbreviated form:

$$
\left\{F_{2 N x 1}\right\}=\left[G_{2 N x 2 N}\right]\left\{d_{2 N x 1}\right\}
$$

The above equation can also be written as

$$
\left[\left[G_{2 N x 2 N}\right]\left[-I_{2 N x 2 N}\right]\right]\left\{\begin{array}{l}
\left\{d_{2 N x 1}\right\} \\
\left\{F_{2 N x 1}\right\}
\end{array}\right\}=\left[0_{2 N x 1}\right]
$$

Equation (43) is a $2 N \times 2 N$ algebraic system with respect to the following $4 N$ unknowns

$$
\begin{aligned}
& \left\{d_{2 N x 1}\right\}=\left\{\begin{array}{lllllllllll}
u_{1} & \vartheta_{1} & u_{2} & \vartheta_{2} & u_{3} & \vartheta_{3} & \cdots & u_{N-1} & \vartheta_{N-1} & u_{N} & \vartheta_{N}
\end{array}\right\}^{T} \\
& \left\{F_{2 N x 1}\right\}=\left\{\begin{array}{lllllllllll}
F_{1} & M_{1} & F_{2} & M_{2} & F_{3} & M_{3} & \cdots & F_{N-1} & M_{N-1} & F_{N} & \left.M_{N}\right\}^{T}
\end{array}\right\}
\end{aligned}
$$

In order for the algebraic system provided by equation (43) to be solvable, it should be completed by $2 N$ more equations. Let's consider the multi-supported pipeline demonstrated in the Figure 2. The boundary conditions for this example are

$$
\left.\begin{array}{l}
u_{1}=0 \\
\vartheta_{1}=0 \\
F_{2}=k u_{2} \\
M_{2}=0 \\
F_{3}=k u_{3} \\
M_{3}=0 \\
\vdots \\
F_{N-1}=k u_{N-1} \\
M_{N-1}=0 \\
u_{N}=0 \\
\vartheta_{N}=0
\end{array}\right\}
$$

The above boundary conditions can be written in the following matrix form:

$$
\left[\left[A_{2 N x 2 N}\right]\left[B_{2 N x 2 N}\right]\right]\left\{\begin{array}{l}
\left\{d_{2 N x 1}\right\} \\
\left\{F_{2 N x 1}\right\}
\end{array}\right\}=\left\{0_{2 N x 1}\right\}
$$

In equation (46) the following is valid: 


$$
\left[A_{2 N x 2 N}\right]=\left[\begin{array}{ccccccccccc}
1 & 0 & 0 & 0 & 0 & 0 & \cdots & 0 & 0 & 0 & 0 \\
0 & 1 & 0 & 0 & 0 & 0 & \cdots & 0 & 0 & 0 & 0 \\
0 & 0 & -k & 0 & 0 & 0 & \cdots & 0 & 0 & 0 & 0 \\
0 & 0 & 0 & 0 & 0 & 0 & \cdots & 0 & 0 & 0 & 0 \\
0 & 0 & 0 & 0 & -k & 0 & \cdots & 0 & 0 & 0 & 0 \\
0 & 0 & 0 & 0 & 0 & 0 & \cdots & 0 & 0 & 0 & 0 \\
\vdots & \vdots & \vdots & \vdots & \vdots & \vdots & \cdots & \vdots & \vdots & \vdots & \vdots \\
0 & 0 & 0 & 0 & 0 & 0 & \cdots & -k & 0 & 0 & 0 \\
0 & 0 & 0 & 0 & 0 & 0 & \cdots & 0 & 0 & 0 & 0 \\
0 & 0 & 0 & 0 & 0 & 0 & \cdots & 0 & 0 & 1 & 0 \\
0 & 0 & 0 & 0 & 0 & 0 & \cdots & 0 & 0 & 0 & 1
\end{array}\right]
$$

Combination of equations (43), (46) yields the following $4 N x 4 N$ algebraic system:

$$
\left[\begin{array}{ll}
{\left[G_{2 N x 2 N}\right]} & {\left[-I_{2 N x 2 N}\right]} \\
{\left[A_{2 N x 2 N}\right]} & {\left[B_{2 N x 2 N}\right]}
\end{array}\right]\left\{\begin{array}{l}
\left\{d_{2 N x 1}\right\} \\
\left\{F_{2 N x 1}\right\}
\end{array}\right\}=\left\{0_{4 N x 4 N}\right\}
$$

The condition for non-trivial solution of the equation (49) is:

$$
\operatorname{det}\left[\begin{array}{ll}
{\left[G_{2 N x 2 N}\right]} & {\left[-I_{2 N x 2 N}\right]} \\
{\left[A_{2 N x 2 N}\right]} & {\left[B_{2 N x 2 N}\right]}
\end{array}\right]=0
$$

The above equation can be solved numerically, providing the values of $\omega$ versus the values of $U$. Critical are the values of $U$ yielding the transition of $\operatorname{Im}\{\omega\}$ from a positive value (stability) to a negative one (instability). 


\section{Implementation in a representative example}

An S-Glass/Epoxy pipeline is resting on one linear spring located at the middle of its length and on its fixed ends (Figure 3 ). The interior diameter of the pipeline is

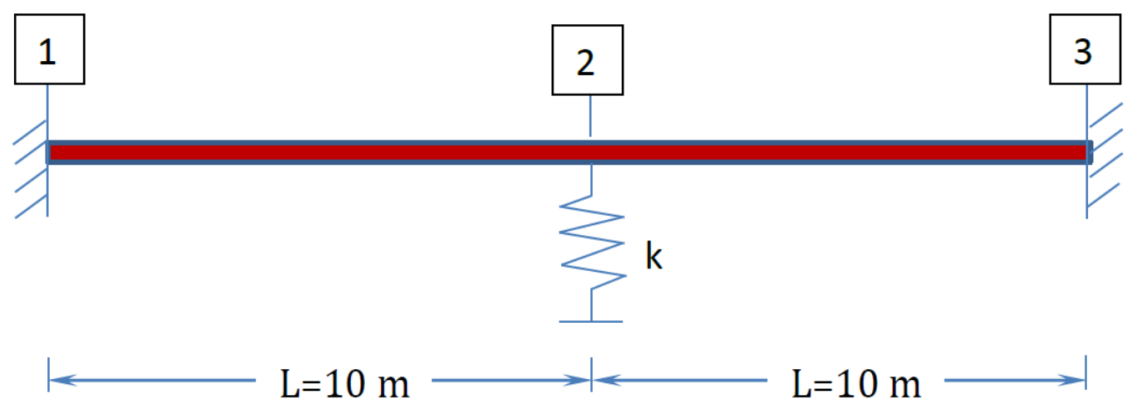

Figure 3: S-Glass/epoxy filament wound pipeline resting on a linear spring.

$\mathrm{D}=0.10 \mathrm{~m}$ and the laminated wall is composed of $\mathrm{N}=50$ layers with thickness $0.15 \mathrm{~mm}$. The material properties in the principal directions of each layer are $\mathrm{E} 1=39 \mathrm{GPa}, \mathrm{E} 2=8.6 \mathrm{GPa}, \mathrm{G} 12=3.8 \mathrm{GPa}, v 12=0.2$, and the masses per unit length $\mathrm{m}$ and $\mathrm{M}$ have values $\mathrm{m}=4.94 \mathrm{Kg} / \mathrm{m}$ and $\mathrm{M}=7.85 \mathrm{Kg} / \mathrm{m}$. With the aid of the procedure described above the effect on the value of critical flow velocity of the span between supports, fiber orientation and value of springs elastic constant is going to be estimated. Taking into account the material data, the [abd] matrix given in equation (2) can be initially obtained for $\vartheta= \pm 30,45,60 \mathrm{deg}$. Then, the parameter Seq can be calculated by the equation (21), yielding $\operatorname{Seq}\left(30^{\circ}\right)=314237 \mathrm{Nm}^{2}$, $\operatorname{Seq}\left(45^{\circ}\right)=196224 \mathrm{Nm}^{2}$, and $\operatorname{Seq}\left(60^{\circ}\right)=146115 \mathrm{Nm}^{2}$. The vectors $\{\mathrm{d}\}$ and $\{\mathrm{F}\}$ contain the following nodal displacements and forces corresponding to the example of Figure 3:

$$
\begin{gathered}
\{d\}=\left\{\begin{array}{llllll}
u_{1} & \vartheta_{1} & u_{2} & \vartheta_{2} & u_{3} & \vartheta_{3}
\end{array}\right\} \\
\{F\}=\left\{\begin{array}{llllll}
F_{1} & M_{1} & F_{2} & M_{2} & F_{3} & M_{3}
\end{array}\right\}
\end{gathered}
$$

The boundary conditions are incorporated in the matrices $[A]$ and $[B]$ :

$$
[A]=\left[\begin{array}{cccccc}
1 & 0 & 0 & 0 & 0 & 0 \\
0 & 1 & 0 & 0 & 0 & 0 \\
0 & 0 & -k & 0 & 0 & 0 \\
0 & 0 & 0 & 0 & 0 & 0 \\
0 & 0 & 0 & 0 & 1 & 0 \\
0 & 0 & 0 & 0 & 0 & 1
\end{array}\right]
$$




$$
[B]=\left[\begin{array}{llllll}
0 & 0 & 0 & 0 & 0 & 0 \\
0 & 0 & 0 & 0 & 0 & 0 \\
0 & 0 & 1 & 0 & 0 & 0 \\
0 & 0 & 0 & 1 & 0 & 0 \\
0 & 0 & 0 & 0 & 0 & 0 \\
0 & 0 & 0 & 0 & 0 & 0
\end{array}\right]
$$

The matrix $[G]$ can be obtained by the implementation of equation (41) to the considered example. Therefore, its members contain functions of the variables $U$ and $\omega$. Using the matrices $[A],[B],[G]$, the equation (50) can be derived yielding a correlation of $U$ versus $\omega$. For four values of spring elastic constant, namely $k=35,45,55,65 \mathrm{KN} / \mathrm{m}$, the equation (50) is solved numerically in order to calculate the eigen-frequencies $\omega$ for three values of fiber orientation, $\vartheta= \pm 30,45$, $60 \mathrm{deg}$, and for three values of pipe span, $L=10,15,20 \mathrm{~m}$ for each value of $\vartheta$.

Starting from a small initial value $U=1.0 \mathrm{~m} / \mathrm{s}$ for the flow velocity which is changed incrementally with step $1.0 \mathrm{~m} / \mathrm{s}$, the eigen-frequency $\omega$ is calculated using the standard commercial program Mathematica ${ }^{\circledR}$. The critical flow velocity values corresponding to the transition of $\operatorname{Im}\{\omega\}$ from a positive value (stability) to a negative one (instability) are demonstrated in the Figures 4(a-c). It should be noted that the numerical results indicated many changes of $\operatorname{Im}\{\omega\}$ from positive to negative values. Therefore, the pipeline has more than one critical flow velocity. In the Figures $4(\mathrm{a}-\mathrm{c})$ only the first critical flow velocity is adopted. According to the above results the following main conclusions can be drawn.

For each value of fiber orientation angle, short pipe segments increases the value of critical flow velocity, improving thus the stability of pipeline. The percentage of $U_{c r}$ shift is higher for the smallest value of $\vartheta$ and decreases progressively for the higher values of $\vartheta$.

(a) For each value of span length $L$, higher values of critical fluid velocities appeared for the smaller values of $\vartheta$. This result was expected since small fiber orientation angles improve the bending stiffness of the pipeline, reducing thus the bending deformation.

(b) For the larger values of $\vartheta$ and $L$, the critical flow velocity seems to be independent of the elastic modulus of the spring support. Therefore, stiff supports do not affect the stability of flexible pipelines. However, for pipelines with higher bending stiffness (e.g. $\vartheta=30 \mathrm{deg})$, stiff elastic springs $(k=35 \div 45$ $\mathrm{KN} / \mathrm{m}$ ) seems to increase the critical flow velocity.

\section{Conclusions}

1. A finite element methodology for critical flow velocity yielding flow-induced instability in FRP filament wound pipelines has been proposed.

2. The exhibited procedure takes into account the fiber orientation, wall thickness, elastic modulus of spring supports, and flow parameters. 


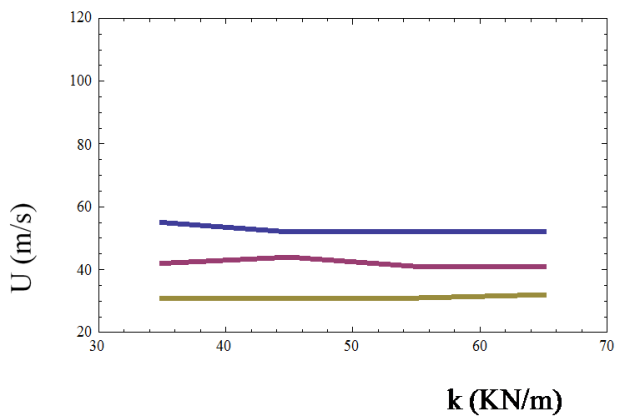

(a)

fiber orientation : $60 \mathrm{Deg}$

- $10 \mathrm{~m}$

- $15 \mathrm{~m}$

$\cdot 20 \mathrm{~m}$

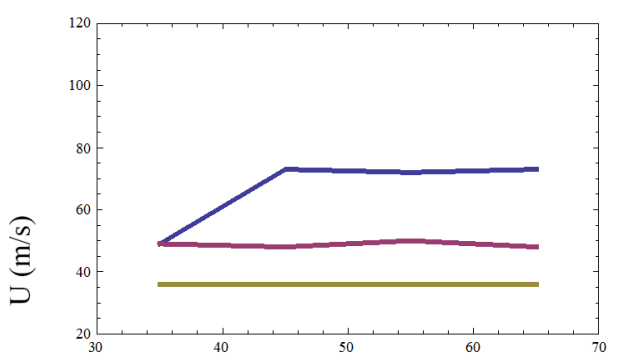

(b)

fiber orientation : 45 Deg

- $10 \mathrm{~m}$

- 15.0

- $20 \mathrm{~m}$

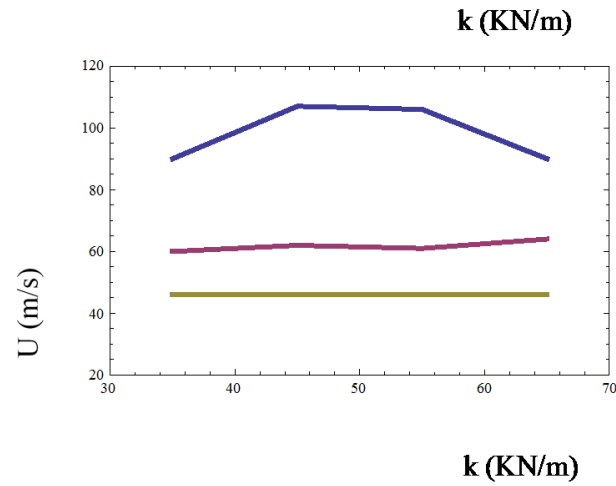

(c)

fiber orientation : $30 \mathrm{Deg}$

- $10 \mathrm{~m}$

- $15 \mathrm{~m}$

- $20 \mathrm{~m}$

Figure 4: Critical flow velocity versus spring elastic constant for $\mathrm{L}=10 \mathrm{~m}, \mathrm{~L}=15$ $\mathrm{m}$, and $\mathrm{L}=20 \mathrm{~m}$. (a) Fiber orientation angle $\vartheta= \pm 60 \mathrm{deg}$, (b) Fiber orientation angle $\vartheta= \pm 45 \mathrm{deg}$, (c) Fiber orientation angle $\vartheta= \pm 30 \mathrm{deg}$

3. The condition for non-trivial solution of the derived pipeline equation yields the eigen-frequencies $\omega$ versus flow velocity $U$. The value of $U$ causing transition of $\operatorname{Im}\{\omega\}$ from a positive to a negative value, defines the boundary between pipes stability and instability.

4. An implementation of the methodology to a simple pipeline resting on an elastic spring, indicated that flow-induced instability can be avoided by short pipeline spans and small fiber orientation angles. Very flexible pipelines cannot be affected by very stiff supports, while stiff pipelines can improve their stability for certain values of stiff supports. 


\section{References}

[1] Tiratsoo, J., Editorial, Journal of Pipeline engineering, 12(6), 2013.

[2] Kollár, L.P., Springer, G.S., Mechanics of composite structures, Cambridge University Press: Cambridge, 2009.

[3] Pavlou, D.G., Composite materials in piping applications, Destech Publications: Lancaster, 2013.

[4] Zaitsev, V.F., Polyanin, A.D., Handbook of exact solutions for ordinary differential equations, CRC Press; Florida 2003. 